\title{
Silver Nanoparticles as Nanofungicide and Plant Growth Promoter: Evidences from Morphological and Chlorophyll 'a' Fluorescence Analysis
}

\author{
Manisha Bawskar', Sunita Bansod ${ }^{1}$, Dnyaneshwar Rathod ${ }^{1}$, \\ Carolina Alves dos Santos ${ }^{2}$, Pramod Ingle ${ }^{1}$, Mahendra Rai ${ }^{1}$, Aniket Gade ${ }^{1, *, \text { (D) }}$
}

\begin{abstract}
Fungal infections are major issue in agricultural crop plants that affects the growth of plant causing huge economic losses. Silver nanoparticles (AgNPs) have shown antimicrobial effects. Here, we report the potential application of phytostabilized AgNPs as a novel nanofungicide and growth promoter in Vigna radiata (L.) R. Wilczek (Mung). The aqueous extract of Azadirachta indica (Neem) challenged with $\mathrm{AgNO}_{3}(1 \mathrm{mM})$ develops a brown colour, indicating AgNPs synthesis. Detection of AgNPs was monitored by UV-Vis spectroscopy, which showed peak at $415 \mathrm{~nm}$. Further confirmation was done by Fourier Transform Infra-Red Spectroscopy which illustrates functional groups present in the capping proteins. Nanoparticle Tracking Analysis, and Transmission Electron Microscopy confirmed the synthesis of spherical, polydispersed nanoparticles in the range of 15-35 nm. AgNPs proved to exhibit antifungal potential in suppression of fungal plant pathogens. Seed germination percentage was much higher for the AgNPs treated seeds as compared to control. Handy-PEA analyzer (measures chl-a fluorescence) indicating seedlings inoculated with AgNPs were grown as the healthy plant with greater vitality, compared to control plants. Phytostabilized AgNPs were found to be biocompatible and have a promising attribute in developing a potent nanofungicide to enhance productivity rate and prevent fungal infections in crops with no potential toxicity.
\end{abstract}

\section{Introduction}

The use of modern techniques for monitoring crops and fields to optimize plant growth is essential for smart agricultural practices. Common issues like fungal and insect infections, drought, exposure to harsh weather conditions, and soil salinity affect growth of plants that lead to huge economic losses. For the large-scale crop production, farmers use chemical fertilizers, pesticides and fungicides to control the insect and fungal infections. Excessive use of these chemicals leads to major environmental and health hazards due to their non-biodegradable nature and biomagnification [1]. Hence, the current trend in agriculture research is aimed either at managing fertilizer's use to reduce environmental problem or to search for environment friendly fertilizer. From ancient period, silver is well known for its strong broad spectrum antimicrobial activity against plant

\footnotetext{
${ }^{1}$ Nanobiotechnology Lab, Department of Biotechnology, Sant Gadge Baba Amravati University, Maharashtra, Amravati 444602, India

${ }^{2}$ LaBNUS - Biomaterials and Nanotechnology Laboratory,

University of Sorocaba, Sorocaba/SP, Brazil

${ }^{*}$ Corresponding author:

E-mail: aniketgade@sgbau.ac.in; aniketgade@gmail.com

Tel.: (+91) 9422164349, 9822114906
}

DOI: 10.5185/aml.2021.15702 pathogens through its multiple mode of inhibitory action [24], therefore may serve as alternative for control of various plant pathogens over synthetic fungicides [5-7]. This has aroused an interest in metal nanoparticles or a combination of two or more metal nanoparticles, which can be used to control various plant pathogenic microbes. Nanotechnological approaches in plants reduce the chances of disease and pest incidence thus aids in efficient crop production. Nanoparticles mediate nutritional transport, increase insect resistance and suppress fungal infections, which can make AgNPs, a possible antimicrobial and a plant growth promoter that can be used in agriculture. Nanoformulated agrochemicals can be used to mediate genetic transformation and develop disease resistance in plants. For the effective use of silver in control of plant diseases include the primary knowledge on the antifungal activity of various silver compounds against plant pathogens and development of effective biocidal agent to increase the disease suppression efficacy $[\mathbf{3 , 8 , 9}]$.

Nanotechnology includes synthesis, characterization, manipulation, fabrication, exploration and application of nanosized (1-100 nm) materials for the development of science and technology. The field of nanotechnology is interdisciplinary and fascinating research area in modern material science concerned with the development of nanodevices and nanomaterials, which can impart novel applications in plant biotechnology and agricultural research [10]. They can also be used for enzymatic nano 
bioprocessing for the conversion of agricultural and food wastes into energy and other useful by-products [11].

In the recent years, several methodologies have been developed in the field of nanotechnology to have control on shape and size of nanoparticles $[12,13 a, 14]$. It's now easy to synthesize nanoparticles of particular shape and size according to specific requirements. New application of nanoparticles and nanomaterials is increasing rapidly as nanotechnology helps in overcoming the limitation of size. It also deals with the materials whose structure exhibit significantly novel and improved physicochemical and biological properties due to their nano size. Nanoparticles have a small size but larger surface area to the volume ratio as compared to macro-sized materials. These improved properties are determined by size, shape, composition, concentration, crystallinity and morphology, which are different from bulk form of the same material making them so commercially desirable [13b]. Due to antifungal activity, silver can be potentially utilized in management of plant diseases $[3,15,16]$. Until now, there are a few research evidences that proved applicability of silver as an effective control measure against plant pathogens $[\mathbf{1 7 - 2 0}]$. The treatment of AgNPs has been reported to increase in ascorbate and chlorophyll content in leaves of asparagus [21]. Broad spectrum antimicrobial activity of AgNPs was reported against spore producing fungal pathogens [17]. A significant reduction in mycelial growth was observed for the spores incubated with AgNPs [22]. To have effective control, the biocidal AgNPs can be applied well before the penetration and colonization of fungal spores within the plant tissues. Inhibitory effect of AgNPs was also reported against Powdery mildew on cucumber and pumpkin [18]. Even there are reports of potent inhibitory effects of biosynthesized AgNPs against different fungal plant pathogens $[\mathbf{2 3 , 2 4}]$. A little contribution has been made to elucidate the exact mechanism by which AgNPs affect the uptake, translocation and growth of plants. The efficacy of AgNPs was also studied in extending the vase life of gerbera flowers [25], and in inhibition of microbial growth and reduced vascular blockage [26], which increases water uptake that maintains the turgidity of gerbera flowers. Thus, specifically an infected tissue alone can be targeted to develop novel nanoparticle delivery systems. Hence, nanoparticulate formulations proved to be novel agrochemicals with high specificity and improved functions. Other reports also have revealed a positive influence of AgNPs in the plant growth promotion in Brassica juncea [27], Phytolacca americana [28], Phaseolus vulgaris [29], and Zea mays [29]. It has also been reported that nanoparticle treatment could induce higher chlorophyll contents in Asparagus and Sorghum $[\mathbf{2 1 , 3 0 ]}$. The effect of AgNPs on seed germination was also reported, that demonstrated improvement in plant growth and productivity $[\mathbf{3 1}, \mathbf{3 2}]$.

In the present study, we have studied phytosynthesis (synthesis using plant) of AgNPs by Azadirachta indica. The advantages of using plants for synthesis of nanoparticle is their easy availability, safe to handle, easy biomass handling and possess a diverse metabolites that can acts as a reducing and stabilizing agent. These synthesized nanoparticles were characterized and evaluated for their growth promoting activity with antifungal efficacy against plant pathogenic fungi. Plant growth parameters of $V$. radiata such as percent of seed germination, shoot length, root length, chlorophyll ' $a$ ' fluorescence were studied. Excellent growth promoting activity was demonstrated with the aid of morphological and physiological markers using Handy PEA analyzer.

\section{Experimental}

All chemicals including silver nitrate, antifungal discs, amphotericin-B antibiotic were purchased from Hi-Media, India.

\section{Synthesis of AgNPs by leaf extract of Azadirachta indica}

Twenty-gram fresh leaves of $A$. indica were collected, washed with $70 \%$ alcohol for $10 \mathrm{~min}$ followed by sterile distilled water. Finely cut leaves were boiled in $100 \mathrm{ml}$ sterile distilled water and extract was obtained by filtration through Whatman filter paper No.1. Thus, extract collected was used for the synthesis of AgNPs.

\section{Characterization of AgNPs}

\section{Visual detection}

The $1 \%$ leaf extract was challenged with $1 \mathrm{mM} \mathrm{AgNO}_{3}$ for the synthesis of AgNPs. The yellowish-green color changes to dark-brown clearly indicate the formation of AgNPs. Color change was developed immediately after the treatment of freshly prepared plant extract with $1 \mathrm{mM}$ $\mathrm{AgNO}_{3}$. The synthesized AgNPs were then purified and concentrated by repeated centrifugation at $15000 \mathrm{~g}$ for 30 min to remove unreacted silver ions and metabolites from the plant extract. The pellet was collected and dissolved in deionized water.

\section{$U V$-Visible spectrophotometer}

The treated plant extract was scanned in the wavelength ranging from $200-800 \mathrm{~nm}$ at a resolution of $1 \mathrm{~nm}$ by UVvisible spectrophotometer (Shimadzu 1700, Japan).

\section{Nanoparticle Tracking Analysis (NTA)}

Nanosight (LM-20, UK) was used to determine the average size of the synthesized AgNPs by diluting $5 \mu 1$ of sample with nuclease free water. Diluted sample was injected into the sample chamber and observed through LM 20 and the size was measured.

\section{Fourier Transform Infra-Red Spectroscopy analysis (FTIR)}

Synthesized AgNPs were dried in a hot air oven and mixed with $\mathrm{KBr}$, crushed using mortar and pestle and then were subjected to FTIR (Perkin-Elmer FTIR-1600, USA) analysis by scanning the spectrum in the range of 400-4000 $\mathrm{cm}^{-1}$ at a resolution of $4 \mathrm{~cm}^{-1}$. 


\section{Zeta potential analysis}

The zeta potential was measured by Malvern Zeta Sizer 90 (ZS-90) to determine the stability of nanoparticles. $30 \mu \mathrm{L}$ of nanoparticle sample was diluted and sonicated for $15 \mathrm{~min}$ at $20 \mathrm{~Hz}$. Then mixture was filtered with the help of 0.22 $\mu \mathrm{m}$ filter and used for zeta potential measurement.

\section{Transmission electron microscopy}

The size and morphology of AgNPs were examined with a JEOL JEM-1011 Transmission Electron Microscope (TEM) operating at an acceleration voltage of $100 \mathrm{kV}$. Specimen for TEM observations were prepared by pipetting a few drops of nanoparticle dispersed solution onto a carbon-coated $\mathrm{Cu}$ mesh grid.

\section{Antifungal activity}

The antifungal activity of the AgNPs was evaluated against plant pathogenic fungi including Fusarium oxysporum (MTCC 1755), Fusarium graminearum (MTCC 1893), Phoma glomerata (MTCC 2710) and Aspergillus niger (MTCC 41) on potato dextrose agar by disc diffusion method. The antifungal activity was determined by growing fungi on potato dextrose agar plates. Four discs impregnated with $20 \mu \mathrm{L}$ AgNPs $(1 \mathrm{mg} / \mathrm{ml})$, antibiotic (Amphotericin-B) and $1 \mathrm{mM} \mathrm{AgNO}_{3}$, Antibiotic+AgNPs respectively were placed onto the agar plate inoculated prior with the test fungi and incubated overnight at $25^{\circ} \mathrm{c}$. The zone of inhibition was measured after $72 \mathrm{~h}$.

\section{Assessment of plant growth promotion activity of AgNPs}

\section{Treatment of Vigna radiata with AgNPs}

Seeds of $V$. radiata (Mung) were collected from local market of Amravati. Seeds were washed with $1 \% \mathrm{H}_{2} \mathrm{O}_{2}$ for 5 minutes followed by $70 \%$ alcohol for 2 minutes. Finally, the seeds were rinsed with sterile distilled water thrice. Some seeds were soaked in distilled water which were taken as a control, and others were in AgNPs solution $(1 \mathrm{mg} / \mathrm{ml})$ for $2 \mathrm{~h}$ followed by rinsing with distilled water twice and placing on a germinating paper in a petri dish. After germination of three days, the seedlings were transferred to a pot containing 3:1 ratio of soil and sand under natural light condition. The experiment was performed in triplicate. Plants were regularly watered and examined for their morphological characteristics. After 2-3 days of germination, percent of seed germination and shoot lengths were recorded at periodic time interval. Percent germination was calculated by dividing the number of seeds germinated over the total number of seeds inoculated and expressed as percentage (\%).

\section{Measurement of Chlorophyll 'a' fluorescence}

The chlorophyll ' $a$ ' fluorescence measurement was carried out using Handy PEA (Plant Efficiency Analyzer) (Hansatech Instrument Pvt Ltd, UK). Both experimental and control set were made in triplicate and were subjected to measure Chlorophyll ' $a$ ' fluorescence. Plants were placed in dark at room temperature for $15 \mathrm{~min}$ before measurement. The dark-adapted samples were illuminated homogenously over an area of $4 \mathrm{~mm}$ diameter with three light-emitting diodes (LEDs). The induced fluorescence was quickly measured. Initially, observations were recorded at $10 \mu$ s intervals for the first $300 \mu \mathrm{s}$. The Handy PEA data was analyzed by JIP-test to calculate the specific and phenomenological fluxes using Biolyzer software [3335]. The Handy PEA data was used to analyze the vitality and photosynthetic competence of experimental plants against control.

\section{Height, Fresh Weight/Dry Weight of Shoot and Roots}

After twenty days of inoculation, the phenotypical characters such as height, length of roots, fresh and dry weight of both shoots and roots were studied.

\section{Results and discussion}

In the present study, the plant growth promotion activity of phytosynthesized AgNPs was evaluated. A. indica plant extract was used as reducing and stabilizing agent for the synthesis of AgNPs because it is readily available and also showed potent antimicrobial activity against bacteria and fungi [36]. On treatment with $1 \mathrm{mM} \mathrm{AgNO}_{3}$, the colour of the plant filtrate was changed to dark brown indicating the synthesis of phytostabilized AgNPs (Fig. 1).
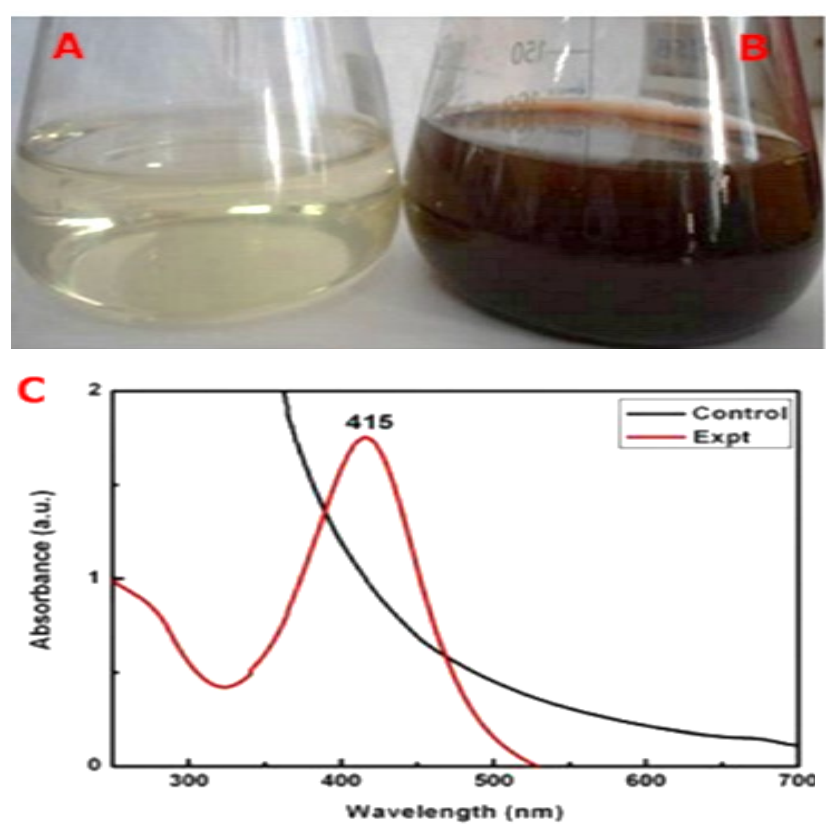

Fig. 1. Synthesis of AgNPs using neem extract [A-Control (Neem filtrate); B-Experimental (AgNPs)], $\mathrm{C}=\mathrm{UV}-\mathrm{Vis}$ spectra of phytosynthesized AgNPs.

The synthesized AgNPs were scanned in the range of 200-800 nm of UV-Vis spectrophotometer and observed a sharp narrow peak with a maximum absorbance at $415 \mathrm{~nm}$. This sharp peak indicates that, nanoparticles were spherical in nature. The absorbance provides information regarding 


\section{Advanced Materials Letters https://aml.iaamonline.org}

the size and structure of the nanoparticles based on their surface plasmon resonance [37].

These phytostabilized AgNPs were concentrated and purified for further characterization. Nanoparticle tracking analysis (NTA) reveals that the nanoparticles were having average size $88 \mathrm{~nm}$ with a standard deviation (SD) of 44, and concentration as $3.20 \times 10^{8}$ particles $/ \mathrm{ml}$ (Fig. 2(a)). The PDI value ranges from 0.0 (for a perfectly uniform sample with respect to the particle size) to 1.0 (for a highly polydispersed sample with multiple particle size populations). The PDI value for AgNPs was found to be 0.458 which is found within a range of 0 to 1 . This value does show polydisperse nature of the synthesized nanoparticles.

Zeta potential is a measure of the electrostatic potential on the surface of the nanoparticles and is related to the electrophoretic mobility and stability of the suspension of nanoparticles [38]. On the basis of zeta potential value of the nanosilver suspension, one can determine whether or not the AgNPs will form aggregate and potentially settle out of suspension. The zeta potential of the phytosynthesized nanosilver suspension measured by Malvern Zeta sizer 90 (ZS 90, USA) was found to be -35.6 which denotes its stability (Fig. 2(b)).

The higher the absolute value of the zeta potential, the more likely the nanoparticles will remain in suspension; whereas, lower the magnitude of the zeta potential, the more likely the nanoparticles will form aggregate together [38]. Gade and colleagues have reported that the value above 30 $\mathrm{mV}$ irrespective charge on the nanoparticle is stable [39].

FTIR analysis confirmed that silver ions were reduced and stabilized by the capping agent (Fig. 2(c)). FTIR analysis reveals the presence of specific functional groups constituting organic molecules. The present FTIR spectra showed absorption peaks to be associated with the stretching vibrations for $-\mathrm{C}-\mathrm{H}\left(2935 \mathrm{~cm}^{-1}\right), \mathrm{N}-\mathrm{H}$ bend $\left(1625 \mathrm{~cm}^{-1}\right)$, -C-N- stretch $\left(1025 \mathrm{~cm}^{-1}\right)$ that corresponds to amide linkages of the protein $[\mathbf{4 0 , 4 1 ]}$. This confirms the association between proteins or other biomolecules i.e., secondary metabolites, free amino groups or cysteine residues present in the proteins, which have the strongest ability to bind silver metal.

The major chemical constituents present in the neem extract were identified as nimbin, nimbidin, quercetin, azadirachtin. These compounds are known to possess potent insecticidal activity, and antimicrobial activity against bacteria and fungi which are now getting resistant to antibiotics $[\mathbf{4 2 , 4 3}$. Several groups reported phytochemicals and heterocyclic compounds as capping agent for nanoparticles [44] and combination of AgNPs and natural compounds with antimicrobial properties Francine [45]. Gade and colleagues reported the ability of quercetin a quinine compound in the reduction of silver ions to form AgNPs [39]. Hence, these properties were exploited to develop potent antifungal agent which will eventually promote plant growth by suppressing fungal infections. The size and topology of synthesized AgNPs was confirmed as uniform, almost spherical and with a size range of 15-35 $\mathrm{nm}$ on the basis of TEM analysis (Fig. 2(d)). The difference in size determined by TEM and NTA is due to the fact that TEM gives the exact size and image of the metal nanoparticles as the beam of electrons used in it transmits through an ultrathin specimen, which interacts with the specimen. While NTA calculates hydrodynamic radius, which was always larger. There are some reports which proves the fact that NTA analysis results in larger particle sizes compared with TEM [46].
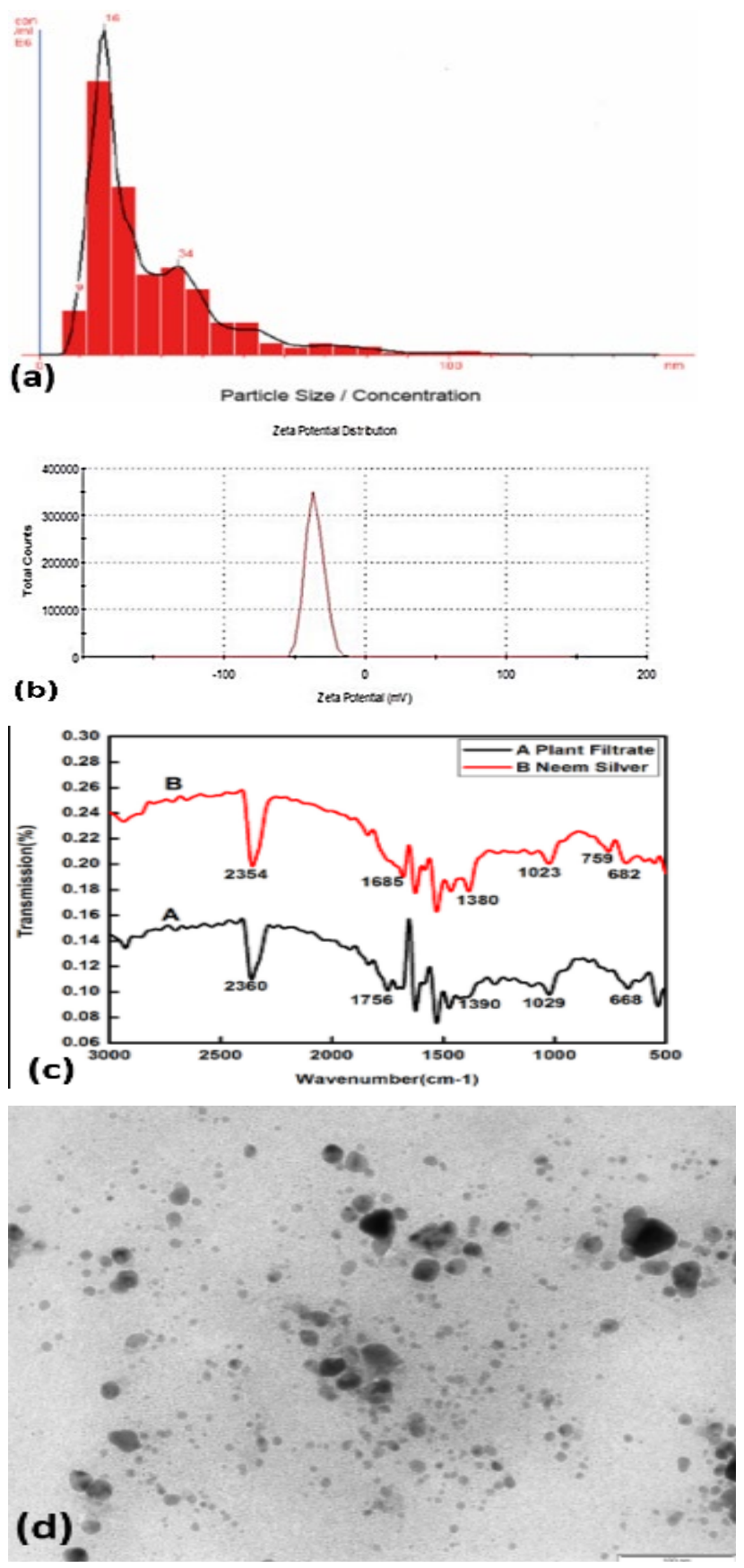

Fig. 2 (a) Nanoparticle Tracking Analysis of Phytosynthesized AgNPs (LM 20); (b) Zeta potential analysis of Phytosynthesized AgNPs; (c) FTIR analysis of phytosynthesized AgNPs where, A-Plant filtrate, B-Neem Silver; (d) TEM analysis of phytosynthesized AgNPs shows uniform, spherical with a size ranging from $5-25 \mathrm{~nm}$. 


\section{Advanced Materials Letters https://aml.iaamonline.org}

\section{Evaluation of antifungal activity}

The purified biogenic AgNPs were evaluated for antifungal activity against plant pathogenic $F$. oxysporum, $F$. graminearum, $P$. glomerata and $A$. niger. The antifungal activity was determined by disc diffusion method. Four discs viz., AgNPs, Amphotericin-B, $1 \mathrm{mM} \mathrm{AgNO}_{3}$ and AgNPs+Antibiotic discs were placed on to the medium. AgNPs exhibited excellent antifungal activity singly as well as when used in combination with antibiotic against Fusarium species followed by $A$. niger and then $P$. glomerata proving it as a novel antifungal agent (Fig. 3(a)).

The average zones of inhibition (ZOI) shown by AgNPs only, did not show much variation $(13-16 \mathrm{~mm}$ ) for all four fungus. In the similar way, precursor $\mathrm{AgNO}_{3}$ even smaller ZOIs than that of the AgNPs (i.e., 10-13 mm). antibiotic Amphotericin-B alone showed significant ZOI ranging between 15-18 mm. But, when AgNPs were combined with antibiotic the fungicidal activity of AgNPs was enhanced to a greater extent. This indicates that the test materials followed same order of fungicidal activity as, $\mathrm{AgNPs}+\mathrm{Ab}>\mathrm{Ab}>\mathrm{AgNPs}>\mathrm{AgNO}_{3}$. The results suggest that AgNPs are capable of inhibiting these test pathogens, however, results vary according to the concentration and type of AgNPs applied to pathogens [3]. Previous reports of mechanism of inhibition suggests that AgNPs may directly bind to and penetrate the cell membrane to kill spores and can significantly inhibit mycelial growth according to the concentration of the applied nanoparticles $[47,48]$. Although the penetration of AgNPs into microbial cell membranes is not yet completely understood [49]. In addition, AgNPs exert detrimental effect on several biomolecules including sugar, protein, n-acetyl glucosamine and lipid of culture filtrate and cell wall components of both plant pathogens [50]. The combined effect of a standard antifungal agent (fluconazole) with AgNPs was reported against pathogenic plant and human fungal pathogens [51]. Similar antimicrobial potential of AgNPs in combination with antibiotics was demonstrated against $P$. acnes, $C$. albicans, T. mentagrophytes and $M$. canis [52].

\section{Evaluation of plant growth promotion activity of AgNPs}

Activity of AgNPs was evaluated for their plant growth promotion and seed germination activity on Mung bean plant. The seeds treated with AgNPs were found to be with higher germination percentage $(93.33 \%)$ as compared to untreated (Control) (60\%). Mazumdar and Ahmed claimed that different concentrations of AgNPs have different effect on seed germinations, root and shoot growth [53]. In the present study, the seeds treated with AgNPs $(1 \mathrm{mg} / \mathrm{ml})$ showed higher seed germination $(93.33 \%)$ as compared to control $(60 \%)$. After 20 days, difference between root and shoot height, their fresh and dry weight biomass was calculated. Ultimately the length of root and shoot of $V$. radiata treated plants was greater as compared with control (Fig. 3(b)).
The plants treated with AgNPs showed greater fresh and dry weight and length of roots and shoots as compared to control which is due to the remarkable influence of AgNPs treatment (Fig. 3(c)).

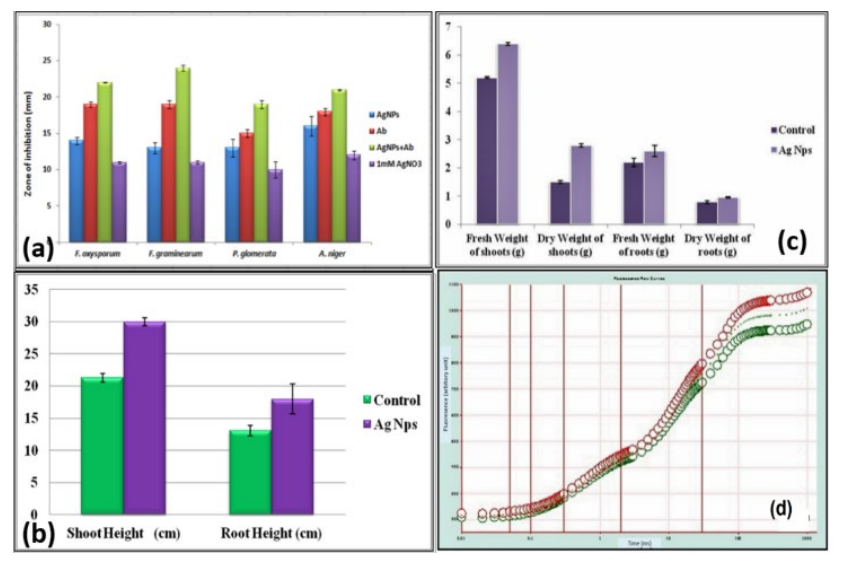

Fig. 3. (a) Graphical representation of Antifungal activity of phytosynthesized AgNPs against test fungi; (b) Difference between shoot and root height; (c) Difference between the fresh weight and dry weight of Shoots and Roots; (d) Fluorescenc e plot shows Chlorophyll a fluorescence difference between control and experimental (Green = Control, Red $=$ Experimental).

\section{Analysis of Chlorophyll 'a' fluorescence}

The study of chlorophyll 'a' fluorescence has been considered to calculate the specific energy fluxes (ABS/RC, ETo/RC, TRo/RC and $\mathrm{DIo} / \mathrm{RC}$ ), phenomenological fluxes (ABS/CS, ETo/Cs TRo/CS, $\mathrm{DIo} / \mathrm{CS}, \mathrm{RC} / \mathrm{CS}$, $\mathrm{ABS} / \mathrm{CS}$ ) and PIabs. The plants treated with AgNPs showed maximum $\mathrm{Chl}$ 'a' fluorescence as compared to the control (Fig. 3(d)). Due to application of AgNPs the treated plants showed highest Chl ' $a$ ' fluorescence than that of control. It is concluded that AgNPs might act as catalyst for growth promotion activity. The radar graph shows the average OJIP test values resulting from the $\mathrm{Chl}$ ' $\mathrm{a}$ ' fluorescence measurement of control and treated plants with AgNPs. The plants treated with AgNPs showed the highest performance index per absorption (PIabs) and less dissipation per reaction centre (DIo/RC) as compared to control (Fig. 4).

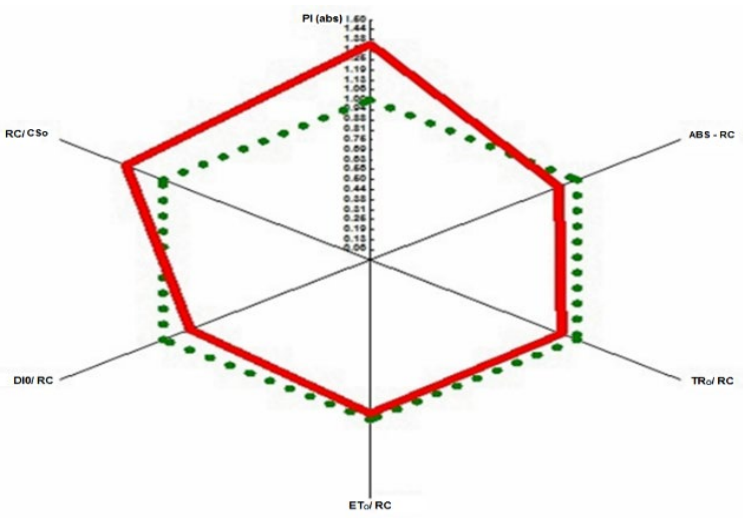

Fig. 4. Radar plot of experimental (Red) and control (Green) plants. 

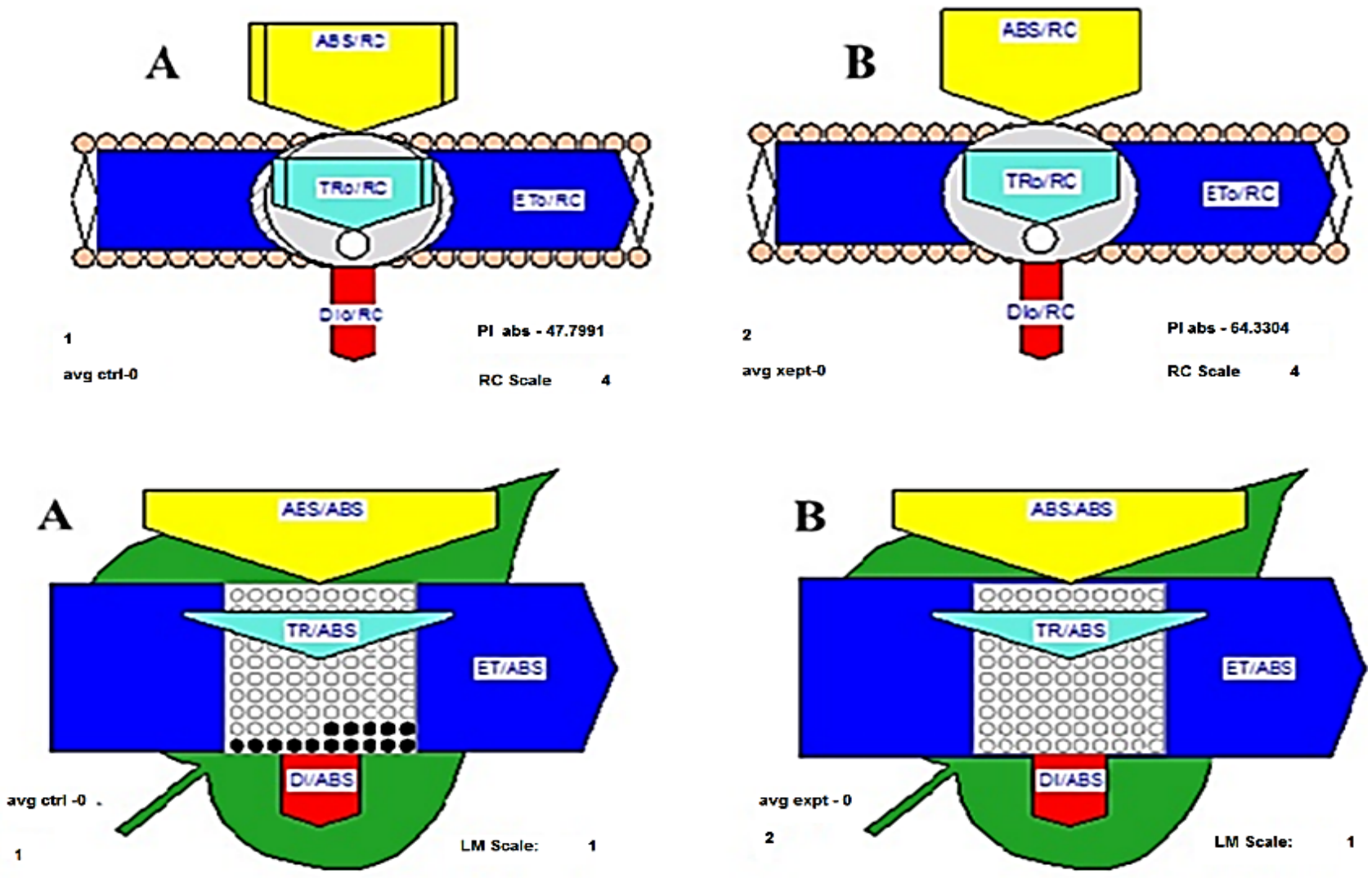

Fig. 5. Pipeline model of control and experimental showed membrane and leaf model (Leaf model).

Moreover, the plants treated with AgNPs showed higher number of reaction centre per cross section ( $\mathrm{RC} / \mathrm{CSo})$, electron trapping per reaction centre $(\mathrm{ETo} / \mathrm{RC})$ as compared to the control.

In the pipeline model the membrane model of treated plants showed higher performance index per absorption (PIabs) (64.3304) and less dissipation per reaction centre (DIo/RC) as compared to control (47.7991) (Fig. 5).

These parameters also provide evidence that the AgNPs accelerate the growth of treated plants as compared to control. The black points in leaf model represent the fraction of inactive reaction center. The treated plants do not show any fraction of inactive reaction centers but control showed the fraction of inactive reaction center. Phenomenological leaf models are based on calculations of parameters per leaf cross section based on equations derived by Strasser and coworkers for control and experimental plants [54]. In leaf model the thickness of each arrow shows the value of absorbance (ABS/RC), trapping per absorbance (TR/ABS), electron transport per absorbance (ET/ABS) or dissipation per absorbance (DI/ABS). This is all for leaf cross section. The $\mathrm{RC} / \mathrm{ABS}$ and $\mathrm{ETo} / \mathrm{ABS}$ were found to be increased in plants treated with AgNPs as compared to control plants. All these changes may be due to less deactivation of $\mathrm{RC}$ in treated plants than control which cause perturbation in electron transfer (ETo), so that the number of active RC controlled the intensity of the photosynthetic reactions.

\section{Conclusion}

The Phytosynthesized and phytostabilized AgNPs were found to be helpful in overall growth profile of $V$. radiata. The present findings revealed that these AgNPs enhanced plant growth promotion with a broad spectrum antifungal activity against selected plant pathogenic Fusarium, Phoma and Aspergillus species. Plants treated with AgNPs had shown maximum root and shoot length as compared to the control plants. Further, thorough field trials are needed in order to use AgNPs for better crop yield.

\section{Acknowledgement}

We would like to express our sincere thanks to Department of Science and Technology, Government of India, New Delhi for providing financial support under Nanomission program (No. SR/NM/NS-96/2010 (C) DST New Delhi).

\section{Conflict of interest}

There are no conflicts to declare.

\section{Keywords}

Phytosynthesized AgNPs, plant growth promoter, nanofungicide, HandyPEA analyzer. 
Received: 18 April 2021

Revised: 13 July 2021

Accepted: 15 July 2021

\section{References}

1. Ali, H.; Khan, E.; Hindawi. J. Chem. 2019.

2. Clement, J.L.; Jarret, P.S.; Metal-Based Drugs. 1994, 467.

3. Kim, S.W.; Jung, J.H.; Lamsal, K.; Kim, Y.S.; Min, J.S.; Lee, Y.S.; Mycobiology. 2012, 53 .

4. Bhaskar, B.; Ahammed, S.K.; Chaitanya, B.H.; Rasheed, V.A.; Prasad, T.N.V.K.V; Int. J. Res. App. Nat. Soc. Sci. 2016, 2347; ISSN (E): 2321-8851, 4(10) 105-114.

5. Park, H.J.; Kim, S.H.; Kim, H.J.; Choi, S.H.; Plant. Pathol. J. 2006, 295.

6. Abd-Elsalam, K.A.; Alghuthaymi, M.A.; J. Nanotech. Mater. Sci. 2015, 1 .

7. Worrall, E.A.; Hamid, A.; Mody, K.T.; Mitter, N.; Pappu, H.R.; Agronomy, 2018, 285.

8. Alghuthaymi, M.A.; Almoammar, H.; Rai, M.; Said-Galiev, E.; Abd-Elsalam, K.A.; Biotechnol. Biotechnol. Equip. 2015, 221.

9. Brauer, V.S.; Rezende, C.P.; Pessoni, A.M.; De Paula, R.G.; Rangappa, K.S.; Nayaka, S.C.; Gupta, V.K.; Almeida, F.; Biomolecules. 2019, 521.

10. Scrinis, G.; Lyons, K.; Int. J. Sociol. Food. Agric. 2007, 22-44.

11. Carmen, I.U.; Chithra, P.; Huang, Q.; Takhistov, P.; Liu, S.; Kokini, J.L.; Food Technol. 2003, 24.

12. Narmadha, E.; Hemashenpagam, N.; Sathiya, V.S.; Vasantha Raj, S.; Int. Res. J. Pharm. 2013, 166

13. (a) Santos, C.A.; Ingle, A.; Seckler, M.M.; Rai, M.; IET Nanobiotechnology. 2016, 1.

(b) Santos, C.A.; Seckler, M.M.; Balcao, V.; Chaud, M.V.; Rai, M.; Vila, M.C.; IET Nanobiotechnology. 2016, 11.

14. Varanda, L.C.; Souza, C.G.S.; Moraes, D.A.; Neves, H.R.; Souza Jr, J.B.; Silva, M.F. et al. Acad. Bras. Ciênc. 2019, e20181180. https://doi.org/10.1590/0001-765201920181180.

15. Mishra, S.; Singh, B.R.; Singh, A.; Keswani, C.; Naqvi, A.H.; Singh, H.B.; Plos One, c97881, 2014.

16. Mishra, S.; Singh, H.B.; Appl. Microbiol. Biotechnol. 2015.

17. Jo, Y.K.1; Kim, B.H.; Jung, G.; Plant. Dis. 2009, 1037.

18. Lamsal, K.; Kim, S.; Jung, J.H.; Kim, Y.S.; Kim, K.S.; Lee, Y.S.; Mycobiology. 2011, 26.

19. Al-Othman, M.R.; Abd El-Alsalaam, K.; Mahmoud, E.; El-Shikha, M.; Digest. J. Nanomaterials. Biostructures. 2014, 151.

20. Lee, S.H.; Jun, B.H.; Int. J. Mol. Sci. 2019, 865.

21. An, J.; Zhang, M.; Wang, S.; Tang, J.; LWT-Food Sci. Technol. 2008, 1100 .

22. Marek, J.K.; Magdalena, K.; Anna, G.; Canadian J. Microbiol. 2010, 247.

23. Krishnaraj, C.; Ramachandran, R.; Mohan, K.; Kalaichelvan, P.T.; Spectrochim. Acta. Part A Mol. Biomol. Spectrosc. 2012, 95-99.

24. Gopinath, V.; Velusamy, P.; Spectrochim. Acta. Part A Mol. Biomol. Spectrosc. 2013, 170.

25. Solgi, M.; Kafi, M.; Taghavi, T.S.; Naderi, R.; Postharvest. Biol. Technol. 2009, 155.

26. Liu, J.; He, S.; Zhang, Z.; Cao, J.; Lv, P.; He, S.; Cheng, G.; Joyce, D.; Postharvest. Biol. Technol. 2009, 59.

27. Sharma, P.; Bhatt, D.; Zaidi, M.G.H.; Saradhi, P.P.; Khanna, P.K.; Arora, S.; Appl. Biochem. Biotechnol. 2012, 2225.

28. Yin, L.; Colman, B.P.; McGill, B.M.; Wright, J.P.; Bernhardt, E.S.; PLOS ONE. 2012, e47674.

29. Salama, H.M.H.; Int. Res. J. Biotech. 2012, 190.

30. Namasivayam, S.K.R.; Chitrakala, K.; J. Biopesticides. 2011, 97

31. Savithramma, N.; Ankanna, S.; Bhumi, G.; Nano Vision. 2012, 2.

32. Singh, A.K.; Rathod, V.; Singh, D.; Mathew, J.; Kulkarni, P.; Int. J. Res. Stud. Agri. Sci. 2016, 6.

33. Rodriguez, R.M.; Avlov, S.; Gonzalez, A.; Oukarroum, A.; Strasser, R.J.; Environ. Chem. Lett. 2003, 201.

34. Repkova, J.; Brestic, M.; Zivcak, M.; Cereal. Res. Commun. 2008, 1747.

35. Zivcak, M.; Brestic, M.; Olsovska, K.; Slamka, P.; Plant Soil Environ. 2008, 133.
36. Alavijeh, P.K.; Alavijeh, P.K.; Sharma, D.; Asian J. Plant. Sci. Res. 2012, 496.

37. Elechiguerra, J.L.; Burt, J.L.; Morones, J.R.; et al, J. Nanobiotechnol. 2005, 1.

38. Dougherty, G.M.; Rose, K.A.; Tok, J.B.; Pannu, S.S.; Chuang, F.Y.; Sha, M.Y.; Chakarova, G.; Penn, S.G.; Electrophoresis. 2008, 1131.

39. Gade, A.K.; Gaikwad, S.C.; Tiwari, V.; Yadav, A.; Ingle, A.P.; Rai, M.K.; Curr. Nanosci. 2010, 370.

40. Goormaghtigh, E.; Ruysschaert, J.; Raussens, V.; Biophys. J. 2006, 2946.

41. Adochitei, A.; Drochioiu, G.; Revue. Roumaine. de Chimie. 2011 783.

42. Shankar, S.S.; Rai, A.; Ahmad, A.; Sastry, M.; J. Colloid. Interf. Sci. 2004, 496.

43. Tripathy, A.; Raichur, A.M.; Chandrasekaran, N.; Prathna, T.C.; Mukherjee, A.; J. Nanopart. Res. 2010, 237.

44. Iravani, S.; Green Chem. 2011, 2638.

45. Francine, A.T.; Chaud, M.V.; Grotto, D.; Jozala, A.F.; Pandit, R.; Rai, M.; Dos Santos, C.A.; Aaps. Pharmscitech. 2017, 1.

46. Gade, A.; Gaikwad, S.; Duran, N.; Rai, M.; Biotechnol. Appl. Biochem. 2013, 482.

47. Min, J.S.; Kim, K.S.; Kim, S.W.; Jung, J.H.; Lamsal, K.; Kim, S.B.; Jung, M.; Lee, Y.S.; Plant. Pathol. J. 2009, 376.

48. Aguilar-Mendez, M.A.; Martin-Martinez, E.S.; Ortega-Arroyo, L.; Cobian-Portillo, G.; Sanchez-Espindola, E.; J. Nanopart. Res. 2011, 2525 .

49. Hwang, E.T.; Lee, J.H.; Chae, Y.J.; Kim, Y.S.; Kim, B.C.; Sang, B.I.; Gu, M.B.; Small. 2008, 746.

50. Ouda, S.M. Res. J. Microbiol. 2014, 34.

51. Gajbhiye, M.; Kesharwani, K.; Ingle, A.; Gade, A.; Rai, M.; Nanomed. Nanotechnol. 2009, 382

52. Gupta, A.; Bonde, S.; Gaikwad, S.; Ingle, A.; Gade, A.; Rai, M.; IET Nanobiotechnol. 2013, DOI: 10.1049/iet-nbt.2013.0015.

53. Mazumdar, H.; Ahmed, G.U.; Int. J. ChemTech. 2011, 1494.

54. Strasser, R.J.; Srivastava, A.; Tsimilli-Michael, M. The fluorescence transient as a tool to characterize and screen photosynthetic samples, Yunus M, Pathre U, Mohanty P (Eds); Taylor \& Francis, London. 2000

\section{Authors biography}

Dr. Manisha Bawskar: She received her PhD from SGBA University, Maharashtra India. Her expertise is on metal nanoparticles biosynthesis and characterization. Also, contributed many research articles, reviews and book chapters of international repute. Dr. Sunita Bansod: She received her PhD from SGBA University, Maharashtra India. Her expertise is on metal nanoparticles phytosynthesis and characterization. Also, contributed many research articles, reviews and book chapters of international repute.

Dr. Dnyaneshwar Rathod: He received her $\mathrm{PhD}$ from SGBA University, Maharashtra India. Her expertise is on metal nanoparticles mycosynthesis and characterization. Also, contributed many research articles, reviews and book chapters of international repute.

Dr. Carolina Alves dos Santos: She earned a PhD from University of São Paulo at School of Pharmaceutical Sciences-Brazil (2011); and post doctorate at Polytechnic School in University of São Paulo-Brazil (2014). Presently she is a Researcher at the University of Sorocaba-Brazil (2016). she has experience in the field of Pharmacy, with emphasis in Pharmaceutical Technology, working mainly on drug stability, biosensors, validation and development of analytical methodologies, nanotecnhology and nanobiotecnhology.

Mr. Pramod Ingle: Pursuing $\mathrm{PhD}$ in Biotechnology from SGBA University, Maharashtra India under supervision of Dr. AK Gade. His expertise is on metal nanoparticles biosynthesis and characterization. Also, contributed many research articles, reviews and book chapters of international repute.

Prof. Mahendra Rai: is a former Professor and Head at the Department of Biotechnology, Sant Gadge Baba Amravati University at Amravati, Maharashtra, India. His present research interests are nanobiotechnology in medicine, agriculture and bioethanol production. He has published more than 400 research papers in national and international journals. In addition, he has edited/authored more than 40 books and 6 patents.

Dr. Aniket Gade: is an Assistant Professor at the Department of Biotechnology, Sant Gadge Baba Amravati University at Amravati, Maharashtra, India. His present research interests are nanobiotechnology in medicine, agriculture and bioethanol production. He has published more than 104 research publications of national and international repute. 